\title{
The Significant Applications of Temperament in Anxiety and Depression: A Developmentally Contextual Trend
}

\section{Mohammad Qasim Abdullah*}

Clinical Psychology, Faculty of Education, University of Aleppo, Syria

*Corresponding Author: Mohammad Qasim Abdullah, Clinical Psychology, Faculty of Education, University of Aleppo, Syria.

Received: September 16, 2019; Published: October 21, 2019

DOI: 10.31080/ASNE.2019.02.0119

\begin{abstract}
The aim of this article is to investigate the relationship between anxiety and depression. The two states are affective disorders, and It is hypnotized that the temperament is the fundamental of this relationship between these states. The findings indicate, for good understanding, predicting and controlling anxious and depressive behavior require of using multi-methodologies from developmental perspective.
\end{abstract}

Keywords: Anxiety; Depression; Temperament

The term "emotion" refer to more general concept. It is the pattern of behavioral, subjective, cognitive and physiological responses to underlying psychobiological state. "Affect" usually refer to the minute-to-minute or hour-to-hour situation in the individual emotional state, whereas "mood" usually refers to the more enduring aspects of the individual emotional state, particularly the presence of a pervasive emotion, which endures over relatively long periods, because of hereditable basis and construct. The concept of "mood" is closely related to the concept of "temperament" and "character". Thus, we talk about individuals who have depressed mood as a long-standing dysthymic temperament. On the other hand, rapidly fluctuating affect states are often characteristic of certain personality types, notably histrionic personality disorder and borderline personality disorders [1]. The term "affective disorder" popular in clinical and research settings, refer to depression conditions and elated manic states. Affective disorders should include anxiety states as well as abnormalities of other emotions such as guilt, anger and hostility. Neurotic Affective states" has been used to refer to both anxiety and depressive states [2].

The normal emotion in anxiety is fear, while the normal emotion in depression is sadness. Anxiety disorders include disorders that share features of excessive fear and anxiety and related beha- vioral disturbances. Fear is the emotional response to real or perceived imminent threat, whereas anxiety is anticipation of future threat. Obviously, these two states overlap, but they also differ, with fear more often associated with surges of autonomic arousal necessary for fight or flight, thoughts of immediate danger, and escape behaviors, and anxiety more often associated with muscle tension and vigilance in preparation for future danger and cautious or avoidant behaviors. Sometimes the level of fear or anxiety is reduced by pervasive avoidance behaviors. Panic attacks feature prominently within the anxiety disorders as a particular type of fear response. Panic attacks are not limited to anxiety disorders but rather can be seen in other mental disorders as well. (Table 1) includes the normal emotion, and psychopathologic symptoms.

\section{Anxiety disorders in DSM-5}

The anxiety disorders embodied in DSM-5 are: Separation Anxiety Disorder, Selective Mutism, Specific Phobia, Social Anxiety Disorder (Social Phobia), Panic Disorder, Panic Attack Specifier, Agoraphobia, Generalized Anxiety Disorder, Substance/Medication-Induced Anxiety Disorder, Anxiety Disorder Due to Another Medical Condition, Other Specified Anxiety Disorder, Unspecified Anxiety Disorder [3]. 


\begin{tabular}{|c|c|c|}
\hline & Anxiety & Depression \\
\hline Normal emotion & $\begin{array}{ll}\text { - } & \text { Fear } \\
\text { - } & \text { Worry }\end{array}$ & $\begin{array}{ll}\text { - } & \text { Sadness } \\
\text { - } & \text { Disappointment }\end{array}$ \\
\hline $\begin{array}{l}\text { Psychopatholog- } \\
\text { ic symptoms and } \\
\text { syndromes }\end{array}$ & $\begin{array}{l}\text { - } \quad \begin{array}{l}\text { Anxiety } \\
\text { state }\end{array} \\
\text { - } \quad \text { Phobia }\end{array}$ & $\begin{array}{ll}\text { - } & \text { Depressed sate } \\
\text { - } & \text { Demoralization }\end{array}$ \\
\hline
\end{tabular}

Table 1: describes the emotions and their relations with anxiety and depression.

Depression, otherwise known as major depressive disorder or clinical depression is a common and serious mood disorder. Those who suffer from depression experience persistent feelings of sadness and hopelessness and lose interest in activities they once enjoyed. Aside from the emotional problems caused by depression, individuals can also present with a physical symptom such as chronic pain or digestive issues. For diagnosing, depressed symptoms must be present for at least two weeks [4].

\section{Depressive disorder in DSM-5}

The Depression disorders embodied in DSM-5 include: Disruptive Mood Dysregulation Disorder, Major Depressive Disorder, Persistent Depressive Disorder (Dysthymia), Premenstrual Dysphoric Disorder, Substance/Medication-Induced Depressive Disorder, Depressive Disorder Due to Another Medical Condition, Other Specified Depressive Disorder, Unspecified Depressive Disorder, Specifies for Depressive Disorders (APA, 2013) [3] The DSM-5 outlines the following criterion to make a diagnosis of depression. The individual must be experiencing five or more symptoms during the same 2-week period and at least one of the symptoms should be either (1) depressed mood or (2) loss of interest or pleasure.

1. Depressed mood most of the day, nearly every day.

2. Markedly diminished interest or pleasure in all, or almost all, activities most of the day, nearly every day.

3. Significant weight loss when not dieting or weight gain, or decrease or increase in appetite nearly every day.

4. A slowing down of thought and a reduction of physical movement (observable by others, not merely subjective feelings of restlessness or being slowed down).
5. Fatigue or loss of energy nearly every day.

6. Feelings of worthlessness or excessive or inappropriate guilt nearly every day.

7. Diminished ability to think or concentrate, or indecisiveness, nearly every day.

8. Recurrent thoughts of death, recurrent suicidal ideation without a specific plan, or a suicide attempt or a specific plan for committing suicide.

To receive a diagnosis of depression, these symptoms must cause the individual clinically significant distress or impairment in social, occupational, or other important areas of functioning. The symptoms must also not be a result of substance abuse or another medical condition [3]. The controversy has changed significantly from 1980 according to the DSM-3.

Temperament is predisposition for anxiety and depression

The most general definition of temperament is that it consists of biologically rooted individual differences in behavior tendencies that are present early in life and are relatively stable across various kinds of situation and over the course of time (Bates, 1987) The term is most often applied to behavioral qualities of emotion, attention and activity. There are big areas of behaviors. Only an exceedingly grandiose temperament theory would try to encompass them in the whole. The most important aspects allocated to temperament are 1- the positivity vs negativity of emotional response in general, emotional responses to novel stimuli in general, emotional responses to familiar and unfamiliar people in particular., and emotional expression in response to internal state such as hunger and boredom; 2- attentional orientation patterns, such as soot ability when distressed and distractibility of attention; and 3- motor activity's vigor and frequency and appropriate self-modulation of activity. The temperament concept as behavior traits ought to have a clear genetic basis, and there is evidence for an early-appearing genetic component in a number of temperamental variables [5].

Depression has been defined as a state of low mood and aversion to activity, can affect a person's thoughts, behavior, tendencies, feelings, and sense of well-being. Symptoms of the mood disorder is marked by sadness, inactivity, difficulty in thinking and concentration and a significant increase/decrease in appetite and time spent sleeping. A great deal of people also have feelings of dejection, hopelessness, and sometimes-suicidal tendencies. It can be either 
short term or long term depending on the severity of the person condition. A depressed mood is a normal temporary reaction to life events, such as the loss of a loved one. It is also a symptom of some physical diseases and a side effect of some drugs and medical treatments. Depressed mood may also be a symptom of some mood disorders such as major depressive disorder or dysthymia [3].

Personality and temperament influence each other, and the most important aspect of personality are self-concept, defense mechanisms and goodness of fit. In Psychopathological practice, depression is the most important complication of anxiety disorders occurring in 28 to $91 \%$ of patients. The depressive illness occurring in chronically anxious patients has been labeled secondary, both because it occurs after the onset of the anxiety disorder and appears to develop in reaction to serve anxiety. Although the temporal relationship between depression and anxiety has an important bearing in the controversy, most studies have been limited to anxious patients without prior history of depression [6]. The nature of depression occurring in patients with chronic anxiety should help determine whether it is primary or secondary. It is clear that patients who experience secondary depression have a more chronic and severe anxiety disorders. It has been showed that anxiety neurotics with a history of depression had more persistent symptoms and greater social impairment at follow-up. Depression that is secondary tends to be influenced by the course and treatment of the underlying disorder. Depressive illness that occurs in physically ill persons often resolves when that illness is successfully treated [7].

It is widely recognized that there is considerable overlap between anxiety and depression. Clinicians and researchers fifer in their thinking about this relationship. Through the 1960s and 1970s, the controversy was between unitary and dualistic theories. Controversies of the $196 \mathrm{~s}$ and 1970 s were dominated by efforts to interest new findings from psychopharmacology and from the application of multivariate statistics made possible by high-speed electronic computer [1]. The widely accepted etiological hypothesis proposes a co-occurrence between depression and anxiety, since these conditions share several symptoms and causal factors. However, hopelessness appears to play a unique role in this co-occurrence [8].
It is of special importance to understand the mechanisms involved in the development of depression and anxiety, not only because of the high rates of prevalence of these disorders all over the world but also because this co-occurrence often increases the likelihood that adolescents will develop feelings of hopelessness which is known to have links with suicidal ideation and suicidal behaviors [9].

Another important construct, demoralization, was described as different from depression. Individuals who are demoralized are conscious of their own failure and are unable to meet the expectations of others. When their anxiety levels increase, they are likely to develop feelings of hopelessness and a desire to die [10]. The validity of the distinction between depression and demoralization is further supported by research showing that suicidal ideation is differentially associated with hopelessness and depression. Studies in adolescents have also shown that depression and hopelessness are independent predictors of suicidal ideation. The dysregulation of temperament is the fundamental pathology underlying mood disorders, and its presence in individuals reflects an increased predisposition for developing affective disorders. Moreover, specific affective temperament types (depressive, cyclothymic, hyperthymic, irritable, and anxious) have a strong relationship with suicidal behavior and a study regarding affective temperaments in suicide attempters indicated that compared to controls suicide attempters scored significantly higher on four of the five affective temperaments containing a more or less depressive component [11].

In spite of the fact that all the above factors, depression, anxiety, hopelessness, and affective temperaments, are related to suicidal behavior, no study so far investigated the interrelationship between these phenomena in a single framework. Delineating the associations between these phenomena is crucial in understanding how they mediate and influence one another in the development of suicidal behavior, describing possibilities for intervention on multiple levels. The aim of our present study was to explore the association of the five affective temperaments in the Akiskal model (Depressive, Hyperthymic, Cyclothymic, Irritable, and Generalized Anxious) with anxiety, depression, and hopelessness in a sample of adolescents. Our study also extends the model of demoralization by introducing temperament dysregulation, and we propose a two-factor model that explains the relationships between temperament, anxiety, depression, and hopelessness [6,12]. 
In sum, The relationship between anxiety and depression is complex and not fully elucidated. As normal emotion, depression and anxiety appear to have independent evolutionary and neurological basis, although they frequently occur together, particularly in response to aversive life events. Similarly, in many medical diseases and psychiatric disorders, anxiety and depression symptoms are common and often complicate the diagnosis and treatment of the primary medical and psychiatric conditions [13]. We conclude that the temperament is the fundamental construct for good understanding anxiety and depression disorders.

The most widely used psychobiological technique has been to study the symptomatic response to lactate infusion, which seems to have some specificity to panic disorder. Other challenge substances have included carbon dioxide, yohimbine and caffeine; hyperventilation could also be a contributing factor $[14,15]$. The lactate infusion studies provide a means of experimentally inducing the panic attack and if fully validated as its specificity, would provide not only an experimental model for panic disorder, but also would contribute to the validity of separating panic anxiety and form other type of anxiety particularly generalized anxiety from other form of anxiety, particularly generalized anxiety from other affective disorders, notably depression and depressive disorders. Advances in clinical research methodology become more widespread and further clarification of the relationship between anxiety and depression on one hand, and its relation with temperament and personality on the other hand. This study encourages researchers to use multi-methodologies for good understanding, predicting and controlling the anxious and depressive behaviors with connecting to temperament, especially from developmental perspective.

\section{Bibliography}

1. Klerman G. "Relationship between anxiety and depression". In M. Roth, G. Noyes and G. Burrows: Handbook of anxiety". Elsevier New York (1988).

2. Fox NA., et al. "The Biology of temperament: An integrative approach". In: Nelson C, Luciana M, eds. The handbool of developmental cognitive neuroscience. Cambridge, MA: MIT Press (2008): 839-854.

3. APA: American Psychiatric Association. Diagnostic and Statistical Manual of Mental Disorders, Fifth Edition (DSM-5) (2013).
4. Mata DA., et al. "Prevalence of Depression and Depressive Symptoms Among Resident Physicians: A Systematic Review and Meta-analysis". Journal of the American Medical Association 314.22 (2015): 2373-2383.

5. Kohnstamm A and Rothbart M. "Temperament in childhood". John Wiley and Sons: Chichester, New York, Bristbane, Toronto, Singapore (1989).

6. Schmidt P. "Mood, Depression, and Reproductive Hormones in the Menopausal Transition". The American Journal of Medicine 118.12B.12 (2005): 54-58.

7. Roth S., et al. "Handbook of anxiety". Biological, Clinical and Cultural perspectives. Elsevier: Amsterdam-New York-Oxford (1988)

8. Wilson C and Deane F. "Help-negation and suicidal ideation: the role of depression, anxiety and hopelessness". Journal of Youth and Adolescence 39.3 (2010): 291-305.

9. Hankin B Abramson., et al. "A prospective test of the hopelessness theory of depression in adolescence". Cognitive Therapy and Research 25.5 (2001): 607-632.

10. Frank D. "Psychotherapy: the restoration of morale". The American Journal of Psychiatry 131.3 (1974): 271-274.

11. Rothbart K., et al. "Developing mechanisms of temperamental effortful control". Journal of Personalized Medicine 71.6 (2003): 1113-1143.

12. Akiskal H. "The temperamental foundations of affective disorders". In Interpersonal Factors in the Origin and Course of Affective Disorders, C. Mundt, K. Hahlweg, and P. Fiedler, Eds., Gaskell, London, UK. View at Google Scholar (1996).

13. Prior M., et al. "Does shy-inhibited temperament in childhood lead to anxiety problems in adolescence?". Journal of the American Academy of Child and Adolescent Psychiatry 39.4 (2000): 461-468.

14. Rogers Pies Ronald. "General Medical Drugs Associated with Depression”. Psychiatry (Edgmont) 5.12 (2017): 28-41.

15. Figueiredo M. "Depression and demoralization: phenomenologic differences and research perspectives". Comprehensive Psychiatry 34.5 (1993): 308-311.

\section{Volume 2 Issue 11 November 2019 (C) All rights are reserved by Mohammad Qasim Abdullah.}

